
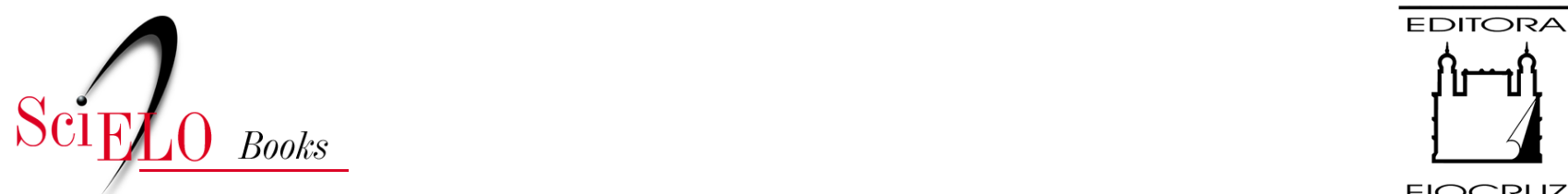

FIOCRUZ

\title{
13. Abordagem avaliativa de uma experiência de promoção da saúde no Rio de Janeiro
}

\author{
Maria de Fátima Lobato Tavares \\ Antônio Ivo de Carvalho \\ Regina Bodstein
}

\section{SciELO Books / SciELO Livros / SciELO Libros}

TAVARES, M.F.L., CARVALHO, A.I., and BODSTEIN, R. Abordagem avaliativa de uma experiência de promoção da saúde no Rio de Janeiro. In: UGÁ, M.A.D., et al., (orgs.). A gestão do SUS no âmbito estadual: o caso do Rio de Janeiro [online]. Rio de Janeiro: Editora FIOCRUZ, 2010, pp. 333-350. ISBN: 978-85-7541-592-4. Available from: doi: 10.7476/9788575415924.0015. Also available in ePUB from: http://books.scielo.org/id/c2hxb/epub/uga-9788575415924.epub.

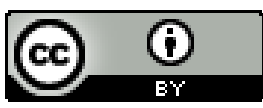

All the contents of this work, except where otherwise noted, is licensed under a Creative Commons Attribution 4.0 International license.

Todo o conteúdo deste trabalho, exceto quando houver ressalva, é publicado sob a licença $\underline{\text { Creative Commons }}$ Atribição 4.0.

Todo el contenido de esta obra, excepto donde se indique lo contrario, está bajo licencia de la licencia $\underline{\text { Creative }}$ Commons Reconocimento 4.0. 


\title{
Abordagem Avaliativa de uma ExperiênCIA de Promoção da Saúde no Rio de Janeiro
}

\author{
Maria de Fátima Lobato Tavares \\ Antônio lvo de Carvalho \\ Regina Bodstein
}

Inspirada no Relatório Lalonde (1974) e consubstanciada na Carta de Ottawa (Brasil/ MS, 2001), a promoção da saúde revitaliza o debate no campo, definindo as condições fundamentais para a saúde (paz, moradia, educação, alimentação, ecossistema estável, recursos sustentáveis, justiça social e equidade). A saúde é vista a partir dos determinantes sociais, culturais, econômicos, educacionais, ambientais e ecológicos e de acesso a bens e serviços essenciais. Esses princípios dão origem a um movimento internacional que se organiza em torno de uma nova agenda que coloca a saúde como um bem público universal, na perspectiva de uma visão sistêmica na qual está em jogo o enfrentamento das desigualdades sociais. São estabelecidas cinco estratégias-chave para a promoção da saúde:

- Políticas públicas orientadas pelos princípios do direito à saúde e à qualidade de vida (intersetorialidade);

- Ambientes saudáveis (sustentabilidade e preservação do meio ambiente);

- Ações comunitárias ('empoderamento' e participação);

- Atuação sobre estilos de vida (considerando os componentes estruturais desses comportamentos ou hábitos);

- Reorientação dos serviços de saúde (desafiando programas e práticas tradicionais no campo da saúde pública).

A promoção da saúde está voltada aos grupos sociais, pois tem por base a questão da saúde/doença na sociedade. Busca formação gerencial e técnica, mas também influenciar outros atores sociais chave, tais como aqueles que têm poder de decisão política, institucional e/ou comunitária. Nesse sentido, profissionais que atuam em promoção da saúde e/ou refletem teoricamente sobre ela lhe outorgam um significado mais amplo, menos centrado nas transformações de condutas individuais e mais orientado para a mudança dos determinantes da saúde em nível macrossocial. 
Converteu-se num conceito unificador para todos aqueles que admitem que, para poder fomentar a saúde, é necessário mudar tanto as condições de vida como a forma de viver.

Hancock (1994) aponta a relação com o poder desde aquele que se estende aos indivíduos e grupos para que sejam capazes de exercer maior controle em suas vidas e sua saúde pessoal e comunitária, até um segundo nível no qual a saúde se estabelece como uma parte vital da agenda política.

Kickbusch $(1996,2007)$ considera que a promoção da saúde trata de um novo tipo de política pública, que tem por base os determinantes da saúde, destacando que a Carta de Ottawa teve por finalidade voltar a situar a saúde da população num ponto central, introduzindo um conceito muito ativo e interativo de saúde que é sua relação com os cenários da vida cotidiana.

Alguns elementos em comum apresentam essas definições analisadas anteriormente:

- Em primeiro lugar, destacam que a promoção da saúde relaciona-se com 'o exercício de um maior controle', isto é, busca que indivíduos e grupos assumam maior consciência em face dos seus direitos e das suas responsabilidades em saúde. No entanto, é importante considerar que só há verdadeiro poder de decisão à medida que haja algum nível de acesso a recursos, que reflita níveis mais altos de empoderamento.

- Outro aspecto é o consenso de que a promoção da saúde 'busca incidir na forma de viver das populações, em seus modos de vida'. Relaciona-se com os hábitos, os costumes, as prioridades, que os indivíduos têm e que estão vinculados ao cuidado com sua saúde física e mental.

- Por último, é feita referência à 'identificação e melhoria nos determinantes da saúde'. Em outras palavras, a promoção da saúde não coloca a responsabilidade do estado de saúde nos sujeitos individualmente, pois considera que este se relaciona com as múltiplas causas que afetam o indivíduo e o coletivo e que é necessário tomar decisões que mudem as condições políticas, econômicas e sociais. Assim, para promover a saúde, é necessário modificar tanto as condições quanto a forma de vida.

Nutbean e Blakey (1996) sintetizam o objeto e os propósitos da promoção da saúde destacando que: ela implica trabalhar 'com' as pessoas, não 'para' as pessoas; começa e acaba na comunidade local; está encaminhada até aos determinantes da saúde, tanto os imediatos quanto os subjacentes; interessa-se tanto pelo indivíduo como pelo meio ambiente; enfatiza as dimensões positivas da saúde; e afeta (e deveria envolver), portanto, todos os setores da sociedade e o meio ambiente.

É indubitável, pois, que a promoção da saúde é uma pedra angular da atenção primária e uma função básica da saúde pública, e nesse sentido tem-se conseguido construir um amplo consenso no que diz respeito à estreita relação existente entre promoção da saúde, saúde e desenvolvimento humano e econômico (WHO, 2008).

Considerando a saúde como um bem e recurso público fundamental e direito humano inalienável, a promoção da saúde tem pautado critérios para as boas práticas 
governamentais e empresariais (Kickbusch, 2004). Dessa forma coloca no centro do debate atual o enfrentamento das iniquidades em saúde, entendidas como diferenças que são não só desnecessárias e evitáveis, mas, sobretudo, injustas (Whitehead, 2000). Trata-se de uma visão dos determinantes como processos sociais, econômicos, políticos e culturais geradores de iniquidades, o que justifica uma profunda reorientação das políticas e intervenções públicas, visando a promover e proteger a saúde e diminuir as desigualdades em saúde.

A carta de Bangkok de 2005 avança nessa direção ao tratar as políticas de promoção da saúde como ações e compromissos em escala local, nacional e global, voltadas para mudanças abrangentes e complexas sobre a qualidade de vida de grupos e comunidades, com ênfase nos "determinantes sociais em saúde" (WHO, 2005). Com base nessa visão ocorre uma revitalização do debate sobre as políticas públicas saudáveis e o fortalecimento dos ambientes favoráveis à saúde. As mudanças pretendidas enfatizam as politicas e ações comunitárias em situações e contextos de iniquidades, gerando propostas de intervenção multiestratégicas e intersetoriais com a mobilização e ampla participação da sociedade civil. Enfim, a base conceitual que sustenta esta nova proposta e um modelo de intervenção, pode ser assim resumida:

- Desigualdades sociais são determinantes para a saúde e a qualidade de vida de indivíduos e comunidades;

- Desigualdades são históricas e socialmente construídas, isto é, têm como princípio o reconhecimento de que as desigualdades são estruturalmente determinadas;

- O modelo proposto é por definição multidisciplinar, multiestratégico e multisetorial, estruturado de forma a mobilizar e promover a participação (from the botton);

- As mudanças pretendidas para enfrentar as desigualdades em saúde extrapolam o âmbito dos sistemas e serviços de saúde;

- O modelo proposto pretende gerar conhecimento científico que pode ser aplicado às políticas públicas e de saúde (evidence based policy).

\section{Promoção da SaÚde no Brasil'}

O esgotamento do paradigma biomédico, a mudança do perfil epidemiológico e os desafios sociopolíticos e culturais das últimas décadas têm ensejado o aparecimento de novas formulações sobre o pensar e o fazer sanitários. Entre estas merecem relevância os paradigmas da saúde coletiva no Brasil e a promoção da saúde, nos países desenvolvidos. Ambos os modelos influenciaram o desenvolvimento do Sistema Único de Saúde (SUS), previsto pela Constituição Federal de 1988 (CF88) e normatizado pelas leis n. 8.080 e n. 8.142 de 1990 (Brasil, 1990a, 1990b), e que foi gestado por um grupo de sanitaristas que afirmava a importância do social na determinação do processo saúde e doença.

\footnotetext{
${ }^{1}$ Esta seção teve por base o artigo de Carvalho, Westphal e Pereira Lima (2007).
} 
Desde meados da década de 1980, vários acontecimentos que se deram no âmbito nacional contribuíram para que a promoção da saúde fosse incorporada como uma nova filosofia e prática nas políticas de saúde.

A Reforma Sanitária brasileira passou a ser amplamente discutida a partir de 1986, logo após a VIII Conferência Nacional de Saúde, que propôs para a sociedade brasileira conceitos e objetivos muito semelhantes aos apresentados na I Conferência Mundial de Promoção da Saúde, realizada em Ottawa, no Canadá, no mesmo ano. Em ambos os documentos - Relatório e Carta de Ottawa - foi definido que a saúde não se reduzia à ausência de doença, mas significava a atenção às necessidades básicas dos seres humanos, em um ambiente que favorecesse seu crescimento e desenvolvimento. Profissionais de saúde envolvidos com o movimento sanitário ocupavam-se naquele momento com a implementação da Reforma Sanitária brasileira ou, mais concretamente, do SUS, idealmente entendido como uma política pública que deveria envolver os vários setores da sociedade (Westphal et al., 2004).

Na prática, os esforços para a implementação do SUS restringiram-se a uma luta do setor saúde para a cura das doenças e, nos últimos tempos, voltada, sobretudo à resolução do financiamento das ações de saúde. Entretanto, se a reforma não concretizou suas propostas iniciais, permitiu uma série de mudanças. Vários progressos foram feitos na perspectiva do SUS: descentralizaram-se as decisões, favorecendo o desenvolvimento de um movimento municipalista de saúde liderado pelos secretários municipais de saúde, fortalecendo a participação e o controle social pela população nas questões de saúde e ampliando conceitos e práticas de saúde.

Outro acontecimento importante deste período foi a Conferência Latino-Americana de Promoção da Saúde, promovida pela Organização Pan-Americana da Saúde (OPS), realizada na Colômbia, em Bogotá, com participação de 550 representantes de 21 países latino-americanos entre eles o Brasil, com a intenção de discutir o significado da promoção da saúde na América Latina e debater princípios, estratégias e compromissos para a melhoria da saúde das populações da região, com vistas à equidade (Buss, 1997).

No ano de 1995, o Conselho Nacional de Secretários Municipais de Saúde (Conasems) se reuniu no Congresso dos Secretários Municipais de Saúde das Américas, em Fortaleza, Ceará. A Carta de Fortaleza, elaborada ao final, expressou publicamente o interesse da sociedade representativa dos secretários municipais de Saúde nas propostas da promoção da saúde. Mencionou as experiências canadenses de Cidades Saudáveis, destacando a possibilidade de transferir progressivamente a ênfase que o Sistema vinha dando na doença para a produção social da qualidade de vida, onde o principal ator deveria ser o cidadão referido ao seu ecossistema.

Outro aspecto foi o fato de a municipalização da saúde fortalecer-se a partir de uma experiência integradora, participativa e criativa, buscando a construção de Cidades Saudáveis (Westphal et al., 2004).

A partir desse momento, várias propostas de implementação do Projeto Cidades Saudáveis começaram a serem incentivadas pela OPS e por técnicos canadenses, e colocadas 
em prática em vários estados do país: Paraná, São Paulo, Rio Grande do Sul, Minas Gerais, Alagoas e outros, com o apoio de importantes segmentos da sociedade, especialmente do Conasems. Também a realização do 'I Fórum Brasileiro de Cidades Saudáveis', no Ceará, em agosto de 1998, foi um apoio a estas iniciativas, chegando mesmo a ser lançada a proposta da Rede Brasileira de Municípios Saudáveis (RBMS) (Westphal, Motta \& Bogus, 1998).

Com um objetivo semelhante, foi formado no início de 1998, na Faculdade de Saúde Pública da Universidade de São Paulo (USP), um grupo interestadual e intersetorial para promover estudos, programas experimentais e intercâmbio de experiências entre cidades que vinham desenvolvendo projetos municipais. Este grupo deu origem ao Centro de Estudos, Pesquisas e Documentação em Cidades Saudáveis (Cepedoc).

Em 1998, o Ministério da Saúde brasileiro passou por uma reformulação estrutural, sendo as ações de promoção da saúde pela primeira vez, oficialmente inseridas na estrutura, alocadas na recém-criada Secretaria de Políticas, que possuía departamentos correspondentes a áreas de formulação, gestão de políticas e avaliação de políticas de saúde. Neste momento e nesta localização na estrutura foi elaborado o Programa 'Assistência Preparatória: o novo modelo de atenção e a promoção da saúde', por meio do qual se firmou uma cooperação internacional do Ministério da Saúde com o Programa das Nações Unidas para o Desenvolvimento (Pnud) e a ajuda da Agência Brasileira de Cooperação (ABC) com vistas ao seu financiamento (Nilson \& Westphal, 1998).

Havia um grande estranhamento e muita resistência dos profissionais de saúde, especialmente os mais ligados ao movimento sanitário e à saúde coletiva, que confundiam o novo modelo de promoção da saúde - apoiada nos princípios da participação dos sujeitos individuais e coletivos nos processos de tomada de decisão, na busca da equidade e na articulação intersetorial para resolver o problema da ampla determinação do processo saúde/doença - com a promoção da saúde funcionalista, primeiro nível da prevenção primária da história natural do processo saúde e doença de Leavell e Clark ou à promoção da saúde behaviorista (Buss, 2003; Carvalho, 2005).

Essa inserção na Secretaria de Políticas colaborou positivamente para a divulgação e o esclarecimento de propostas e para articulação de ações de promoção da saúde a outras políticas e outros programas de saúde, ampliando as oportunidades de seus princípios, valores e suas estratégias a serem inseridos na formulação dessas políticas e desses programas, na sua implementação e avaliação. Neste período, faziam parte da Secretaria de Políticas, instâncias importantes de negociação de pactos entre as esferas de governo com relação à saúde: Comissão Intergestora Tripartite (CIT), Conselho Nacional de Saúde (CNS), Conselho de Secretários Estaduais de Saúde (Conass), Conasems e instâncias de participação de associações profissionais e científicas, que abriram espaço para o esclarecimento do significado da promoção da saúde e de suas possibilidades de contribuição na implantação do SUS.

Dois anos depois, quando muitas experiências e muitos debates haviam sido feitos em várias oportunidades, foi pela primeira vez elaborado um documento básico que propunha a criação de uma Política Nacional de Promoção da Saúde. Esse documento teve o mérito 
de refletir todo o processo que foi sendo construído e articulado entre os diversos atores envolvidos com o tema no país na última década (Brasil/MS, 2002).

Outro evento importante da história da promoção da saúde no Brasil ocorreu em novembro de 2002, com a realização, em São Paulo, da III Conferência Latino-Americana de Promoção da Saúde e Educação em Saúde, uma iniciativa conjunta da União Internacional de Promoção da Saúde e Educação em Saúde, do Ministério da Saúde, da OPS, da Escola Nacional de Saúde Pública Sergio Arouca da Fundação Oswaldo Cruz (Ensp/ Fiocruz) e da USP. A mesma contou com 1.500 participantes que apresentaram seiscentos trabalhos, registrados em Anais, sendo brasileiros a maior parte dos participantes. A grande participação no evento foi uma evidência de que a promoção da saúde já havia formado uma massa crítica significativa no país (Westphal et al., 2004).

Em janeiro de 2003 iniciou-se um novo governo no país e a estrutura do Ministério da Saúde foi reformulada uma vez mais. Os novos dirigentes, muitos deles do movimento da saúde coletiva, novamente mostraram-se resistentes à promoção da saúde, mas, como havia um compromisso internacional de implementar 'um novo modelo de atenção na perspectiva da promoção da saúde', resolveram verticalizá-la, como uma filosofia de atenção, realocando-a na Secretaria Executiva do Ministério da Saúde.

\section{A Consolidação da Promoção da Saúde como Política Nacional de Promoção da SaÚde ${ }^{2}$}

O grupo brasileiro ligado à promoção da saúde constituído por professores de universidades e de centros de formação e gestores de programas continuou a reunir-se no Grupo Temático (GT) de promoção da saúde da Associação Brasileira de Saúde Coletiva (Abrasco), responsável por estudar e oferecer diretrizes ao governo sobre saúde. Várias oficinas envolveram numerosos participantes, precedendo eventos como o Fórum Social Mundial, Congressos da Abrasco, ou no interior das respectivas programações sendo produzidas importantes contribuições para a construção conceitual do campo, compreensão das práticas orientadas pela estratégia de promoção da saúde e para a construção de uma base programática mais consistente e operacional.

Em relatório consolidado em 2004, a promoção da saúde

é uma nova referência paradigmática que, retomando e atualizando a agenda interdisciplinar e intersetorial da Reforma Sanitária, inspira e impulsiona o movimento social orientado para mudanças no campo da saúde, em pelo menos três planos da determinação social do processo saúde e doença:

- plano da atenção à saúde, envolvendo esforços de mudança na lógica assistencial e dos modelos de gestão, na direção de neutralizar a hegemonia medicalizante e favorecer a autonomia dos sujeitos;

- plano da gestão social de políticas públicas, sobretudo no nível local, envolvendo ações intersetoriais nas ações e programas, o combate à fragmentação de políticas,

$\overline{2}$ Esta seção teve por base o artigo de Carvalho, Westphal e Pereira Lima (2007). 
motivando o empoderamento dos indivíduos e grupos, assim como a mobilização e integração de organizações governamentais e não governamentais;

- plano do desenvolvimento geral da sociedade - envolvendo a defesa do social como precedente e impulsionador do econômico, combatendo a primazia do crescimento econômico em detrimento da distribuição equitativa de bem estar na sociedade; 'advocacia' da saúde como componente relevante do complexo produtivo, numa concepção avançada de desenvolvimento social e não como consumidor insaciável de recursos públicos. (Abrasco/GT-PS/Dlis, 2004: 3)

Foi também sugerido que todas as instituições de ensino presentes considerassem como sua responsabilidade impulsionar a pesquisa e a formação em promoção da saúde, tanto nas instituições acadêmicas como nas organizações de gestão em saúde, para fazer avançar os conhecimentos e as práticas dessa disciplina na sua inserção na realidade brasileira (Carvalho, 2006). Concomitantemente e por força das pressões dos participantes do GT de Promoção da Saúde e Desenvolvimento Local Integrado e Sustentável (Dlis) da Abrasco, o Ministério da Saúde não abandonou o tema, promovendo reuniões que focalizaram basicamente a promoção da saúde na atenção à saúde, e integrando-a à Secretaria de Vigilância à Saúde.

Inicialmente a concepção de promoção da saúde mais aceita entre os profissionais de saúde tinha um teor mais comportamentalista, voltado para o controle dos riscos comportamentais, tais como alimentação, uso do tabaco, álcool e drogas e sedentarismo, sem considerar as condições que interferem nas opções pessoais. Entretanto, discussões frequentes para a definição da Política Nacional de Promoção da Saúde possibilitaram a aproximação das concepções dos atores do Ministério da Saúde com as Cartas Internacionais de Promoção da Saúde e os conceitos propostos pelos participantes do GT de Promoção da Saúde e Dlis da Abrasco.

Em 30 de março de 2006, quando foi publicada a Política Nacional de Promoção da Saúde (PNPS), através da portaria n. 687 MS/GM (Brasil/MS, 2006), já estava ratificado o compromisso da atual gestão do Ministério da Saúde com a promoção da saúde e reconhecida a concordância entre os princípios e as diretrizes de ambos. A PNPS tem por objetivo melhorar a qualidade de vida da população e reduzir a vulnerabilidade e os riscos à saúde relacionados aos seus determinantes: modo de vida, condições de trabalho, habitação ambiente, educação, lazer, cultura, acesso a bens e serviços essenciais.

Saúde, no referido documento, é considerada o "resultado dos modos de organização da produção, do trabalho e da sociedade em determinado contexto histórico" (Brasil/MS, 2006: 13). Há o reconhecimento de que o processo saúde/doença está associado a determinantes mais amplos, impossíveis de serem modificados somente pelo aparato biomédico. Essa abordagem pode "favorecer a ampliação de escolhas saudáveis por parte dos sujeitos e das coletividades no território onde vivem e trabalham" (Brasil/MS, 2006: 15).

A promoção da saúde foi considerada como

um mecanismo de fortalecimento e implantação de uma política transversal, integrada e intersetorial, que faça dialogar as diversas áreas do setor sanitário e 
outros setores do governo, o setor privado e não governamental, compondo redes de compromisso e corresponsabilidade quanto à qualidade de vida da população em que todos sejam partícipes na proteção e no cuidado com a vida. (Brasil/MS, 2006: 18)

Pressupõe, portanto, que o setor da saúde, via ações de promoção da saúde, se responsabilize pela execução de ações intersetoriais, uma vez que, para dar conta das determinações sociais dos problemas, são necessárias ações para ampliação de recursos políticos, humanos e financeiros que extrapolam o setor saúde.

Para a consecução dos objetivos da PNPS, de acordo com as suas diretrizes, pode-se identificar dois planos de ação já mencionados que correspondem aos indicados pelo GT da Abrasco, visando ao desenvolvimento das ações de promoção da saúde: atenção à saúde e gestão social de políticas.

Para a implementação da PNPS foi instituído pelo Ministério da Saúde um comitê gestor, do qual fazem parte, representantes de todas as secretarias do Ministério da Saúde, Fundação Nacional de Saúde, Fiocruz, Instituto Nacional do Câncer e da Agência Nacional de Saúde Suplementar, não havendo nenhum membro externo ao setor da saúde. Cabe ao comitê gestor articular dentro do Ministério da Saúde todos os procedimentos voltados para produzir saúde. Para o biênio 2006-2007 foram priorizadas ações voltadas para a divulgação da Política e o estabelecimento de estratégias para o desenvolvimento das ações específicas já mencionadas. O comitê deverá interagir e integrar as três esferas de governo para que as estratégias propostas sejam incluídas no Pacto em Defesa da Vida e se constituam em ações intersetoriais nos estados e municípios, tarefa bastante difícil. Programas de educação permanente para os profissionais de saúde serão uma das estratégias prioritárias incentivadas pelo comitê gestor, para facilitar a implementação da Política.

As estratégias de promoção da saúde no Brasil propõem mudanças em pelo menos três âmbitos, a saber:

- No âmbito do sistema de atenção à saúde, em que se impõem a desmedicalização e a reorientação dos serviços de modo que possam acolher e apoiar indivíduos e grupos na construção da autonomia e do empoderamento vis-à-vis o planejamento e desenvolvimento de ações específicas de promoção da saúde no âmbito da atenção básica e das estratégias de saúde da família;

- No âmbito da gestão e do desenvolvimento local, em que se implementam ações intersetoriais pela saúde e qualidade de vida, como resultado e como motivação para o empowerment das populações e dos sujeitos;

- No âmbito da formulação de políticas e diretrizes gerais para o desenvolvimento sustentável e com qualidade de vida (Carvalho, 2006). 


\section{A Importância das Abordagens Avaliativas em Promoção da Saúde}

O desafio para a promoção da saúde tem sido propor um referencial teórico que reoriente as práticas a partir da articulação entre estilo de vida (habitus, no sentido da concepção de Bourdieu, 1996), processo de implementação participativo de políticas e conhecimento científico aplicado ao campo da saúde. A promoção da saúde, ao propor um modelo abrangente e estrutural (Potvin \& Lessard, 1994; Rütten, 1995; Potvin, Haddad \& Frohlich, 2001; Carvalho et al., 2004), evidencia a crítica ao modelo biomédico e normativo tradicional e a importância das mudanças efetivas e sustentáveis dentro de contextos socialmente definidos.

A promoção da saúde está voltada para um modelo que ultrapasse os fatores de risco atribuídos a comportamentos individuais para incluir no modelo padrões de comportamento que definam estilos de vida relacionados à saúde. Esses padrões são indissociáveis dos contextos e espaços em que as relações sociais ocorrem.

Tendências recentes em avaliação vêm problematizando as intervenções a partir de esquemas analíticos mais abrangentes e da compreensão da natureza sociopolítica dos programas. Está presente a necessidade de identificar elementos estruturantes e que condicionam as intervenções, incluindo o contexto e os cenários, a população-alvo, relações e interesses envolvidos, mecanismos e atividades desencadeadas vis-à-vis conflitos e impasses gerados. A especificidade do contexto que condiciona e explica, em grande parte, a configuração particular e o processo de implementação dos programas é um elemento-chave para esses esquemas. A avaliação trata de analisar os programas a partir da compreensão dos principais atores envolvidos no processo decisório, de seus interesses e de suas racionalidades, vis-à-vis ao contexto altamente reflexivo em que os programas e as avaliações acontecem (Shadish, Cook \& Leviton, 1991).

Para a compreensão das iniciativas participativas e de base comunitária, tal como as intervenções propostas para a melhoria da saúde e da qualidade de vida, modelos abrangentes e reflexivos em relação ao contexto social são fundamentais. $\mathrm{Na}$ medida em que se articulam simultaneamente várias estratégias voltadas para promoção da saúde de uma população definida, têm como ponto de partida o diálogo e a colaboração entre diversos setores governamentais e sociedade civil. A convergência entre as propostas; a interdisciplinaridade das ações; a intersetorialidade e a colaboração; e o interesse da população são elementos-chave (Potvin, Haddad \& Frohlich, 2001; Rootman et al., 2001). Nessa perspectiva, políticas e programas de promoção da saúde preocupam-se com as práticas que se desenvolvem em espaços socialmente definidos, em que demandas econômicas, sociais, culturais, ambientais e políticas emergem e são vocalizadas. Iniciativas mais inovadoras estão preocupadas com as desigualdades em saúde que se manifestam em situações de vulnerabilidade, pobreza e/ou exclusão social.

A avaliação aparece como um componente importante dessas iniciativas. Tem o mérito de trazer uma perspectiva reflexiva e articular pesquisa e processo decisório, na medida em que incita o diálogo e a colaboração entre pesquisadores, gestores, profissionais 
e população envolvida. Desempenha um papel fundamental produzindo conhecimento e informações cruciais para o aprofundamento do diálogo entre todos os envolvidos. A avaliação de iniciativas e ações multiestratégicas, participativas e intersetoriais contribui para o entendimento e o consenso em torno dos objetivos, das atividades e dos resultados que, muitas vezes, são implementados a partir de propostas consistentes e justificáveis eticamente, mas genéricas do ponto de vista operacional, como é o caso das propostas: de 'políticas e municípios saudáveis', 'inclusão social', 'empoderamento', 'desenvolvimento social', melhoria da 'qualidade de vida', fortalecimento do 'capital social', entre outras. As intervenções propostas no âmbito da promoção da saúde articulam quase sempre um conjunto considerável de instituições, setores sociais, parcerias entre níveis distintos de governo e uma diversidade de organizações sociais. Nesse contexto a avaliação ajuda a estruturar e a dar sustentabilidade técnica e política às iniciativas, diante de interesses nem sempre colaborativos.

A efetividade das iniciativas com essas características depende em grande parte, da colaboração e cooperação entre diversos atores (gestores, técnicos e população), mas, sobretudo, da corresponsabilização dos indivíduos e das comunidades. O envolvimento e a adesão no processo decisório em torno das atividades e medidas propostas constituem a base de sustentação dessas ações. As mudanças efetivas são aquelas que reconhecem a diversidade de culturas, hábitos ou estilos de vida e o processo de reconstrução de identidades sociais, do fortalecimento da cidadania e a conformação de redes locais (Pawson, 2003; Sullivan, Barnes \& Matka, 2002). A mobilização está atrelada ao reconhecimento de circuitos positivos de sociabilidade e de confiança preexistentes, isto é, um conjunto de atividades sociorrecreativas, esportivas e culturais da própria comunidade. A presença e a participação comunitária nessas atividades revelam em grande parte o capital social acumulado, constituindo um recurso fundamental para a mobilização e participação em outras iniciativas públicas, mesmo em contextos marcados por exclusão social e ausência de equipamentos urbanos básicos, como foi visto no caso de Manguinhos no Rio de Janeiro (Peres et al., 2005). Identificar a diversidade dos circuitos e das formas de sociabilidade e de reciprocidade - dado seu caráter relacional, mas também crítico e reflexivo - é crucial para as mudanças pretendidas e para o engajamento em iniciativas de mudança social mais abrangente.

Abordagens avaliativas, no caso de propostas de promoção da saúde, contribuem, portanto, para sistematizar, dar visibilidade e consistência ao programa e seus efeitos, já que estamos falando de iniciativas marcadas por um grau considerável de diálogo, colaboração, reflexividade e/ou responsividade ${ }^{3}$ em relação aos atores e ao contexto. Podem ser constituídas de articulações frágeis e improvisadas, sem um planejamento e uma organização que dê suporte, enfim, sem um conjunto de iniciativas que vão sendo desenvolvidas e testadas. Por falta de uma clara teorização (ou pela ausência de um modelo teórico) vis-à-vis

\footnotetext{
3 Responsividade diz respeito ao modo como o desenho do sistema de saúde reconhece e consegue responder às expectativas universalmente legitimadas dos indivíduos em relação aos aspectos não-médicos do cuidado (Blendon et al., 2001).
} 
aos objetivos complexos e às vezes pouco específicos, tais como direitos sociais, cidadania, empoderamento e autonomia, cabe ao avaliador sistematizar e dar sentido a esse conjunto fragmentado de processos, informações e resultados.

Em iniciativas de base comunitária e intersetoriais voltadas para objetivos abrangentes, a abordagem avaliativa ajuda na própria implementação do programa, na medida em que amplia o diálogo e o debate em torno dos objetivos e das propostas. Preocupadas com a melhoria da qualidade de vida e, portanto, frequentemente voltadas para as questões estruturais e para os chamados determinantes sociais da saúde, as iniciativas operam em contextos de profunda desigualdade social e em cenários de extrema pobreza e vulnerabilidade. De tal forma, as abordagens avaliativas, tais como as iniciativas, são reflexivas em relação aos contextos sociais e políticos em que os programas atuam e onde as estratégias participativas e de empoderamento acontecem.

Modelos teóricos são fundamentais para a avaliação de programas tendo em vista que clarificam as conexões das proposições com seus efeitos, e permitem o ajuste entre sua concepção, seus objetivos operacionais e as atividades desenvolvidas. Avançam na compreensão do porquê falham e de como podemos aprender e produzir conhecimento a partir das experiências sejam elas, bem ou malsucedidas (Chen, 1990). A complexidade das intervenções de promoção da saúde desafia a busca de evidências e provas de sua efetividade (Rootman et al., 2001; Potvin et al., 2001; Barnes, Matka \& Sullivan, 2003). Apesar do processo decisório ampliado e do ambiente altamente político e incerto que envolve os programas em seu contexto, quase todos voltados para o combate às desigualdades e/ ou inclusão social e ampliação de direitos, a avaliação se propõe a identificar mecanismos operativos que respondem pelas mudanças e pelos efeitos observados das intervenções. Enfim, estas características exigem um esforço analítico para identificar não só seus principais componentes, mas a chamada 'teoria do programa' que permite relacionar as atividades aos efeitos e às mudanças produzidas.

\section{A Iniciativa de um Programa de Desenvolvimento local Integrado e Sustentável EM Manguinhos (RJ)}

No Brasil, programas de Desenvolvimento Local Integrado e Sustentável (Dlis) foram desenhados em meados dos anos 90, tendo como marco a concepção de desenvolvimento como um processo participativo, equitativo e sustentável (Cepal, 1991) e, portanto, a partir de um conceito de "desenvolvimento local como um processo social que reúne crescimento econômico com redistribuição e melhoria da qualidade de vida da comunidade" (Buss \& Ramos, 2000: 15). Seu processo envolve políticas intersetoriais vis-à-vis organizações de redes de apoio social em um dado espaço socioterritorial.

A ideia incluía os componentes de geração de emprego e renda (microcrédito e cooperativismo), habitação, saneamento, saúde e educação entre outros, a partir de um diagnóstico participativo acerca dos principais problemas e demandas de um território/ comunidade local (Cepal, 1991; Franco, 1998; Buss \& Ramos, 2000). As experiências de 
Dlis na década de 1990 ocorreram em comunidades e municípios pequenos do interior do país, em grande parte, priorizando mecanismos de geração de emprego e renda, por meio de parcerias entre o setor público e privado (Franco, 1998; Buss \& Ramos, 2000; Krutman, 2004). Em meados da década de 1990, a iniciativa ainda era inédita em grandes regiões metropolitanas e em comunidades faveladas, representando um grande desafio para o modelo proposto.

O marco inicial para o programa Dlis/Manguinhos foi o diálogo e a formação, em 1999, de um grupo constituído de representantes de diversas instituições acadêmicas, secretarias estaduais e municipais e de grandes empresas públicas e privadas, liderado pela Ensp/Fiocruz, visando a uma ação integrada para a melhoria da qualidade de vida na região de Manguinhos, onde se localiza a Ensp. Conforme a proposta foi ganhando consistência, o grupo responsável pela coordenação sentiu a necessidade de envolver um grupo de pesquisadores para acompanhar o programa, que desenhou uma proposta de avaliação com profissionais em torno das discussões sobre promoção e desenvolvimento comunitário na região que constitui a área de abrangência do Centro de Saúde Escola da Ensp. O envolvimento e a participação nas reuniões da coordenação do Dlis foi crucial para o acompanhamento do processo decisório, o planejamento das ações e seus desdobramentos para a implantação do programa.

A equipe de avaliação foi integrada ao grupo de coordenação do Dlis, participando como observadora da maioria de suas reuniões e decisões. A perspectiva avaliativa do programa de Manguinhos procurou analisar um modelo de intervenção que tinha como inspiração não só a proposta de Dlis, mas que entendia promoção da saúde de um ponto de vista radical e sistêmico - a partir do enfoque dos determinantes sociais (Ferreira \& Buss, 2002; Carvalho et al., 2004; Tavares et al., 2007) - tendo como principal atribuição monitorar e sistematizar o programa a fim de colaborar na proposta e garantir a sistematização e disseminação da metodologia de implantação do Dlis.

\section{Questões Avaliativas}

A avaliação acompanhou e sistematizou o processo de tomada de decisão que envolvia um considerável conjunto de atores e de propostas intersetoriais, voltados para a mobilização de uma comunidade (ou melhor, um conjunto de 11 comunidades locais) particularmente conturbada como no caso de Manguinhos (Bodstein, Zancan \& Estrada, 2001). Isto é, o grupo responsável pela avaliação estava diante de uma intervenção não só especialmente complexa, com inúmeros atores, conflitos e interesses em jogo, mas que pressupunha o envolvimento e a colaboração ativa de diversos setores do estado, da prefeitura e das empresas da região, mas, sobretudo, a mobilização e participação dos moradores. A perspectiva de se enfocar a avaliação na dinâmica da implantação do programa (Hartz, 1999; Denis \& Champagne, 1997; Rootman et al., 2001), no caso, em um contexto socioterritorial de extrema pobreza e de grande violência, foi fundamental. Estava em jogo uma compreensão de que a avaliação voltada unicamente para buscar resultados e efeitos não era adequada (Chen, 1990; Pawson \& Tilley, 1997), diante de objetivos abrangentes e 
estratégias multifocais em um território extremamente desfavorável, considerando a pouca presença ou ausência quase absoluta do poder público na região. A abordagem avaliativa concentrou-se no que parecia essencial na concepção do programa: sua dinâmica de implantação através de mecanismos e estratégias de mobilização comunitária (fóruns locais) e de pactuação de uma agenda de desenvolvimento multisetorial.

A literatura sobre avaliação da experiência das Health Action Zones (HAZs), experiências desenvolvidas na Inglaterra no final dos anos 90 em áreas urbanas degradadas e socialmente vulneráveis, foi útil na medida em que os princípios e objetivos guardavam semelhanças com a experiência de Manguinhos, apesar da diferença nos níveis de desigualdade social entre os dois países. No caso inglês, diferentemente da proposta de Dlis, a inciativa partiu de uma ação planejada centralmente pelo Ministério da Saúde, visando a uma abordagem sistêmica das desigualdades em saúde, tendo como perspectiva um programa a ser implementado em sete anos, com alocação específica de recursos e com indução da sua execução e posterior avaliação pelo nível central (Sullivan, Barnes \& Matka, 2002; Barnes, Matka \& Sullivan, 2003; Springett, 2005).

$\mathrm{Na}$ perspectiva avaliativa adotada para acompanhar a implantação do Dlis, buscou-se identificar os principais processos e práticas que pudessem dificultar ou impedir que as ações intersetoriais e a participação das comunidades acontecessem. Os níveis crescentes de violência e seu impacto não só na qualidade de vida, mas também no padrão de sociabilidade e colaboração e ação coletiva local foram os principais mecanismos identificados (Jackson et al., 2003, Bodstein \& Zancan, 2003; Peres et al., 2005). Isto é, no contexto de Manguinhos procurou-se analisar processos que historicamente dificultam ações colaborativas, diante de um circuito em que a disputa por espaço e recursos políticos é acirrada e violenta. Assim, o processo avaliativo levou em consideração que os programas sociais são, acima de tudo, sistemas sociais e, mais do que isso, que os programas funcionam à medida que introduzem ideias ou recursos em um conjunto já estruturado de relações sociais (Pawson \& Tilley, 1997; Pawson, 2003; Sullivan, Barnes \& Matka, 2002).

O caráter inovador do programa e os impasses na sua implantação direcionaram a proposta avaliativa para o acompanhamento e a análise dos diversos processos de interlocução que foram construídos nos dois anos e meio de duração do programa. A característica mais marcante do Dlis em Manguinhos foi a dinâmica de interlocução e pactuação em torno do chamado comitê gestor. Este comitê representava um grupo de liderança local, envolvendo pesquisadores, profissionais e técnicos e a administração pública (em seus dois níveis, estadual e municipal), entre setores distintos do governo (habitação, saúde, trabalho, entre outros) e entre governo e sociedade (empresas privadas e organizações sociais presentes na comunidade). A proposta de avaliação do Dlis foi desenvolvida com autonomia, desenhada e redesenhada à medida que a equipe de avaliação atuava de forma integrada ao comitê gestor, participando das reuniões onde as decisões eram tomadas. Dessa forma, foi possível acompanhar e documentar in loco a formação de um espaço decisório, que, pela constituição e representatividade de seus membros, estabeleceu uma articulação e um diálogo inédito para a região. $\mathrm{O}$ fato de a equipe de avaliação ter assento nas reuniões 
periódicas da coordenação do programa permitiu que as inúmeras dificuldades e os inúmeros impasses na implementação das decisões intersetoriais, na integração das agendas e pactuação das ações - tanto no plano governamental como no plano comunitário - fossem documentadas. A abordagem avaliativa se beneficiou tanto do conhecimento sobre a concepção e as principais características do programa como de um conhecimento mais reflexivo, construído por meio da participação no processo de tomada de decisão e no debate das propostas e ações que caracterizavam as reuniões do comitê gestor.

A avaliação esteve centrada nos princípios e objetivos que orientaram a construção do programa, isto é, na visão abrangente que compreende os determinantes sociais, ambientais, culturais, políticos e técnicos da saúde vis-à-vis a análise do contexto em que a proposta estava inserida. Nesse caso, a avaliação se concentra em processos, componentes e mecanismos que são introduzidos em contextos altamente desfavoráveis do ponto de vista da ausência de serviços e equipamentos públicos e do ponto de vista da confiança no poder público. $\mathrm{O}$ conhecimento substantivo sobre os princípios e os objetivos do programa bem como sobre os dilemas para sua implantação motivaram a formulação das questões avaliativas concentradas nas questões-chave que estruturam o modelo teórico da promoção da saúde. Por um lado, o processo de interlocução e pactuação de estratégias intersetoriais que tinham como foco a região de Maguinhos. Por outro, um processo de mobilização comunitária que pressupunha uma estratégia de participação ampliada, via a constituição de fóruns comunitários e o consenso em torno de uma agenda integrada de desenvolvimento local. A avaliação priorizou as dificuldades e os impasses na implantação e na curta duração do programa, isto é, nos desafios de uma proposta em um cenário organizacional e participativo altamente conflituoso e adverso a ações colaborativas. Foi crucial para registrar os conflitos politicos que se acirravam e impediam o aporte de recursos para as ações propostas de habitação, melhoria de infraestrutura urbana e de geração de renda.

As questões avaliativas estiveram voltadas para as dificuldades na interlocução e organização dos moradores e representantes comunitários diante da diversidade e clivagem de interesses locais. O 'contexto do programa' foi analisado como um conjunto estruturado de relações capazes de moldarem práticas sociais e interesses políticos. $\mathrm{O}$ caso permitiu identificar uma clivagem entre grupos locais e associação de moradores, responsável pela falta de diálogo, de confiança e de colaboração em relação ao programa e às mudanças pretendidas. $\mathrm{O}$ uso de metodologia qualitativa, para a compreensão de interesses que modelam práticas e estruturam relações historicamente presentes nas comunidades foi fundamental diante dos objetivos e das questões que atravessaram a implantação do programa. Isto é, no caso do Dlis Manguinhos, havia o conjunto de relações e interesses próprios da esfera intergovernamental, e um espaço definido pelos interesses e pelas clivagens no interior das comunidades, ambos igualmente atravessados por conflitos e contradições, por um ambiente altamente politizado e pouco propício ao consenso e à colaboração, quer no encaminhamento das propostas intersetoriais, quer em relação às demandas e necessidades das comunidades. A falta de integração entre setores e agências governamentais e o fato 
de cada setor ter seus próprios objetivos e interesses foram aspectos que os pesquisadores puderam observar porque estiveram presentes nas reuniões e em todos os fóruns de discussão do programa.

Tanto o processo avaliativo como o diálogo em torno do programa em Manguinhos foram realizados em grande parte com o apoio e a participação ativa de um grupo de moradores, atuando como 'mediadores culturais'. O trabalho de colaboração com esses atores teve início já na fase do diagnóstico participativo da região em que os moradores puderam falar e veicular suas principais preocupações e demandas. Posteriormente, permitiu que o espaço social e os circuitos de solidariedade de Manguinhos fossem mapeados, tendo em vista potencializar e dar visibilidade a ações socioculturais, recreativas, e iniciativas públicas e privadas existentes na região. A identificação e o trabalho junto aos mediadores, todos moradores e todas lideranças locais vinculados a ONGs e ao Centro de Saúde da Ensp, viabilizaram o trabalho de campo em uma região de acesso quase impossível, dados os níveis de violência. Em contrapartida, o processo avaliativo pôde não só reconhecer a importância do trabalho dessas lideranças na preservação de um circuito local de sociabilidade e de solidariedade - tão necessário para a qualidade de vida e para a diminuição da violência local - como também dar visibilidade ao 'capital sociocultural' presente na comunidade.

A avaliação analisou a importância do Centro de Saúde Escola da Ensp/Fiocruz junto à comunidade e ao programa, na medida em que constitui um espaço e serviço extremamente respeitado pelos moradores de Manguinhos, favorecendo a integração de ações e a participação social. A riqueza da avaliação foi não só identificar obstáculos e dificuldades do programa, mas identificar processos virtuosos e potencialidades locais a partir de práticas e de vínculos sociais consolidados. A avaliação da implantação do programa mostrou que iniciativas com as características do Dlis dependem, em grande parte, da ampliação do espaço decisório através de uma dinâmica capaz de mobilizar e valorizar o conjunto das organizações sociais presentes e atuantes no local, mas também de uma nova dinâmica de poder (Bodstein \& Zancan, 2003).

\section{CONCLUSÕES}

Como já mencionamos, saúde é um direito garantido ao cidadão brasileiro pela CF88, que apresenta no capítulo de saúde um conceito amplo, que transcende a ausência de doença e sugere estratégias de recuperação, promoção e proteção da saúde. Apesar da explicitação legal da importância de que o sistema se ocupe desta trilogia de ações, a promoção da saúde continua sendo tratada de forma incipiente no país, tanto dentro do setor da saúde como fora dele, incluindo os meios acadêmicos.

A história da saúde pública brasileira, apresentada aqui, permite compreender alguns dos motivos para uma resistência à adoção da promoção da saúde como um paradigma orientador, como uma estratégia de ação, como princípio orientador de processos de produção social da saúde, conforme definida pela Carta de Ottawa e apresentada no documento da Política Nacional de Promoção da Saúde de 2006. 
Há, realmente, disseminadas na sociedade, várias concepções de promoção da saúde, de caráter comportamentalista ou relacionadas ao modelo explicativo da história natural do processo saúde/doença, que não têm conexão com as concepções e os princípios discutidos e assumidos, por exemplo, pelos participantes do GT de Promoção da Saúde e Dlis da Abrasco ou que perpassam as Cartas das Conferências Internacionais de promoção da saúde e que ainda sejam coerentes com os princípios do SUS.

É fundamental, para que se possa discutir uma PNPS e sua avaliação, que esteja claro qual o referencial teórico no qual o avaliador vai se apoiar para acompanhar, analisar e sugerir modificações nas práticas sobre as quais estará realizando seu estudo.

Em relação à avaliação, aqueles que introduziram a política comentam que, até o momento, o desenvolvimento de estudos para identificar evidências da efetividade da promoção da saúde estiveram vinculados a comportamentos e hábitos dos sujeitos e, portanto, vinculados à concepção comportamentalista da promoção da saúde. As metodologias utilizadas para avaliação da mudança de comportamentos foram estudos epidemiológicos, demonstrativos de associações convincentes entre exposição e doença, ou ainda estudos observacionais prospectivos e até ensaios clínicos randomizados.

Persiste, portanto, o desafio de realizar estudos e pesquisas para avaliar os resultados da PNPS e as ações propostas em sua amplitude, referenciadas nos conceitos e princípios estabelecidos pelo SUS e também indicados na Carta de Ottawa, tais como integralidade, equidade, corresponsabilidade, mobilização e participação social, intersetorialidade, informação, educação, sustentabilidade, entre outros. Persiste o desafio de monitorar e avaliar programas e, mais ainda, de implantar esta política em um país de dimensões continentais e com tantas iniquidades sociais. Para demonstrar que a promoção é efetiva na consecução dos objetivos a que se propõe, é necessário criar estratégias de avaliação que identifiquem resultados apesar da complexidade desta nova proposta e do uso que faz de multimétodos de ação para que sejam verificados os efeitos líquidos de cada um dos métodos e estratégias utilizadas. Só assim será possível colaborar para a formação de sujeitos autônomos capazes de promover mudanças no status quo, interferindo na determinação social do processo saúde/doença.

\section{REFERÊNCIAS}

ABRASCO/GT-PROMOÇÃO DA SAÚDE/DLIS. Promoção da Saúde consolida-se como Grupo Temático da Abrasco e propõe articulação com os demais GTS. In: PRÉ- CONGRESSO DE EPIDEMIOLOGIA, jun. 2004, Recife. Disponível em: 〈www.abrasco.org.br〉.

BARNES, M.; MATKA, E. \& SULLIVAN, H. Evidence, understanding \& complexity - evaluation in non-linear systems. Evaluation, 9(3): 265-284, 2003.

BLENDON, R. J.; KIM, M \& BENSON, J. M. The public versus the World Health Organization on health system performance. Health Affairs, 20(3): 10-20, 2001.

BODSTEIN, R. \& ZANCAN, L. Monitoramento e avaliação do programa de desenvolvimento local integrado e sustentável. The Integrated Local Development Project. Relatório de Pesquisa. Rio de Janeiro, 2003.

BODSTEIN, R.; ZANCAN, L. \& ESTRADA, D. Guia de Equipamentos e Iniciativas Sociais em Manguinhos. Rio de Janeiro: Editora Fiocruz, 2001. 
BOURDIEU, P. Razões Práticas: sobre a teoria da ação. São Paulo: Papirus, 1996.

BRASIL. Constituição da República Federativa do Brasil. Brasília: Senado Federal, 1988.

BRASIL. Lei 8.080 de 19 de setembro de 1990. Brasília: Presidência da República, 1990a.

BRASIL. Lei 8.142 de 28 de dezembro de 1990. Brasília: Presidência da República, 1990b.

BRASIL. Ministério da Saúde. Secretaria de Políticas de Saúde. Projeto Promoção da Saúde: declarações das conferências de promoção da saúde. Brasília: Ministério da Saúde, 2001.

BRASIL. Ministério da Saúde. Política Nacional de Promoção da Saúde: documento para discussão. Brasília: Ministério da Saúde, 2002.

BRASIL. Ministério da Saúde. Portaria n. 687 MS/GM, de 30 mar. 2006. Política nacional de promoção da saúde. Diário Oficial da União, Brasília, 2006. Disponível em: 〈www.saúde.org.br〉.

BUSS, P. M. A Promoção da saúde no desenvolvimento sustentável. In: SEMINÁRIO INSTITUCIONAL DE PROMOÇÃO DE SAÚDE - SESC. I, 2-5 dez. 1997, Rio de Janeiro.

BUSS, P. M. Uma introdução ao conceito de promoção da saúde. In: CZERESNIA, D. \& FREITAS, C. M. F. (Orgs.). Promoção da Saúde: conceitos, reflexões, tendências. Rio de Janeiro: Editora Fiocruz, 2003.

BUSS, P. M. \& RAMOS, C. L. Desenvolvimento local e agenda 21: desafios da cidadania. Cadernos da Oficina Social, (3): 13-65, 2000.

CARVAlHO, A. I. Promoção da saúde: novos rumos da saúde pública. Portal Click Saúde, 7 nov. 2006. Disponível em: <www.ensp.fiocruz.br/informe/INDEX cfm>. Acesso em: 5 mar. 2007.

CARVALHO, A. I.; WESTPHAL, M. F. \& PEREIRA LIMA, V. L. G. Health Promotion in Brazil. Revista Promotion Ė Education, edição especial, 1: 7-12, 2007.

CARVALHO, A. I. et al. Concepções e abordagens na avaliação em promoção da saúde. Revista Ciência E⿱ Saúde Coletiva, 9(3): 521-530, 2004.

CARVAlHO, S. R. Saúde Coletiva e Promoção da Saúde: sujeito e mudança. São Paulo: Hucitec, 2005.

CEPAL. El Desarrollo Sustentable: transformación productiva, equidad y medio ambiente. Santiago do Chile: ONU, 1991. (Publicação da Organização das Nações Unidas n. Doc. LC/G 1648.)

CHEN, H. Theory-Driven Evaluations. London: Sage, 1990.

DENIS, J. L. \& CHAMPAGNE, F. Análise da implantação. In: HARTZ, Z. (Org.). Avaliação em Saúde: dos modelos conceituais à prática na análise da implantação dos programas. Rio de Janeiro: Ensp/Fiocruz, 1997.

FERREIRA, J. R. \& BUSS, P. M. O que o desenvolvimento local tem a ver com a promoção da saúde? In: ZANCAN, L.; BODSTEIN, R. \& MARCONDES, W. B. (Orgs.). Promoção da Saúde como Caminho para o Desenvolvimento Local: a experiência em Manguinhos-RJ. Rio de Janeiro: Abrasco, Fiocruz, 2002.

FRANCO, A de. Desenvolvimento local, integrado e sustentável: dez consensos. Proposta: desenvolvimento local, integrado e sustentável II, 78: 6-19, 1998.

HANCOCK, T. Health promotion in Canada: did we win the battle but lost the war? In: PEDERSON, A.; O’NEILL, M. \& ROOTMAN, I. (Eds.). Health Promotion in Canada. Toronto: W. B. Sauders Canada, 1994.

HARTZ, Z. Pesquisa avaliativa em promoção da saúde. In: BUSS, P. M. (Org.). Promoção da saúde e saúde pública: contribuição para o debate entre as escolas de saúde pública da América Latina. Rio de Janeiro, 1999. (Mimeo.)

JACKSON, S. F. et al. Working with Toronto neighborhoods toward developing indicators of community capacity. Health Promotion International, 18(4): 339-350, 2003.

KIKCBUSCH, I Promoción de la salud: una perspectiva mundial. In: OPS. Promoción de la Salud: una antologia. Washington: OPS, 1996. (Publ. Cient. 557)

KIKCBUSCH, I. The end of public health as we know it: constructing global health in the 21st century. In: INTERNATIONAL CONGRESS ON PUBLIC HEALTH WORLD FEDERATION OF PUBLIC HEALTH ASSOCIATONS, 10, 2004. 
KIKCBUSCH, I. Avanzar hacia una nueva salud pública. Promotion EO Education, 14: 58-59, 2007. KRUTMAN, H. M. Fatores Críticos no Êxito da Gestão de Projetos de Desenvolvimento Local Integrado e Sustentável, 2004. Tese de Doutorado, Rio de Janeiro: COPPE, Universidade Federal do Rio de Janeiro.

LALONDE, M. A New Perspective on the Health of Canadians: a working document. Ottawa: Government of Canada, 1974.

NILSON, E. A. F. \& WESTPHAL, M. F. Country report of Brazil: priorities and major health promotion efforts in 1998-1999. In: WORLD HEALTH ORGANIZATION (WHO). Megacountry Health Promotion Network. Geneva: WHO, 1998. (Annex E: Countries Reports)

NUTBEAM, D. \& BLAKEY, V. El concepto de promoción de la salud y la prevención del Sida. Una base integrada y general para la acción en los años noventa. In: OPS. Promoción de la Salud: una antologia. Washington: OPS, 1996. (Publ. Cient. 557)

PAWSON, R. Nothing as practical as a good theory. Evaluation, 9(4): 471-490, 2003.

PAWSON, R. \& TILLEY, N. Realistic Evaluation. London: Sage, 1997.

PERES, F. et al. Lazer, esporte e cultura na agenda local: a experiência de promoção da saúde em Manguinhos. Revista Ciência $\mathcal{E}$ Saúde Coletiva, 10(3): 757-769, 2005.

POTVIN, L. \& LESSARD, R. Le paradoxe de l'evaluation des programmes communautaires multiples de promotion e la santé. Ruptures, 1: 45-57, 1994.

POTVIN, L.; HADDAD, S. \& FROHLICH, K. Beyond process and outcomes evaluation: a comprehensive approach for evaluating health promotion programmes. In: ROOTMAN, I. et al. (Orgs.). Evaluation in Health Promotion: principles and perspectives. Copenhagen: WHO, 2001. (WHO Regional Publications. European Series, 92)

ROOTMAN, I. et al. (Orgs.). Evaluation in Health Promotion: principles and perspectives. Copenhagen: WHO, 2001. (WHO Regional Publications. European Series, 92)

RÜTTEN, A. The implementation of health promotion: a new structural perspective. Social Science EF Medicine, 41(12): 1.627-1.637, 1995.

SHADISH W. R. J. R.; COOK, T. D. \& LEVITON L. C. Foundations of Program Evaluation: theories of practice. Thousands Oaks: Sage, 1991.

SPRINGETT, J. Geographically - based approaches to the integration of health promotion into health systems: a comparative study of two health action zones in the UK. Promotion $\mathcal{E}$ Education, supl. 3: 39-44, 2005.

SULLIVAN, H.; BARNES, M. \& MATKA, E. Building Collaborative Capacity through 'theories of change' - early lessons from the evaluation of HAZ. England. Evaluation, 8(2): 205-226, 2002.

TAVARES, M. F. L. et al. Analyzing the outcomes of health promotion practices. Promotion $\mathcal{E}$ Education, supl. 1: 21-26, 2007.

WESTPHAL, M. F.; MOTTA, R. M. M. \& BOGUS, C. M. Contribuição para formação de uma rede brasileira de municípios Saludables. Jornal do Conasems, ago. 1998.

WESTPHAL, M. F. et al. A promoción de salud em Brasil. In: ARROYO, H. V. (Org.). La Promoción de la Salud em América Latina: modelos, estructuras y vision crítica. 1. ed. Puerto Rico: Universidad de Puerto Rico, 2004.

WHITEHEAD, M. The Concepts and Principles of Equity and Health. Genova: WHO, 2000.

WORLD ORGANIZATION (WHO). The Bangkok charter for health promotion, in a globalized world, 2005.

WORLD HEALTH ORGANIZATION (WHO). The World Health Report 2008: primary health care now more than ever. Genebra: WHO, 2008. 